\title{
Quantitative In Situ Electrochemical Liquid Cell Characterization of SEI Formation in Lithium Ion Batteries
}

\author{
Robert L. Sacci, Nancy J. Dudney, Karren L. More, and Raymond R. Unocic
}

Materials Science Technology Division

Oak Ridge National Laboratory, Oak Ridge, TN 37831

The increased global demand for alternative electrical energy storage sources has driven the development of lithium ion battery technology for electrical vehicle, grid storage, and portable electronic device applications. In order to develop a fundamental understanding of the electrochemical processes that take place within these energy storage devices, in situ TEM studies need to be conducted simultaneously with accurate quantitative electrochemistry measurements. Specific to Li-ion battery research, the solid electrolyte interphase (SEI) has enabled the development and application of lithium batteries; however, characterizing the formation mechanism and kinetics has remained elusive due to the present lack of high spatial resolution imaging coupled electrochemical characterization. The SEI is a nm-scaled passivating layer that forms locally at the electrode|electrolyte interface as a result of electrolyte decomposition during the initial charging cycle. It then evolves over time during electrochemical cycling [1-2]. The structure, morphology, and physiochemical properties of the SEI are dependent upon a number of factors such as: anion, solvent, electrode material, and the electrochemical cycling conditions. The ongoing formation of radicals (due to the highly negative reduction potential of $\mathrm{Li},-3.04 \mathrm{~V}$ ) leads to disordered SEIs.

Direct visualization of electrochemical processes occurring within commercial Li-ion battery electrolytes requires the use of a closed cell approach. Electron transparent SiN viewing membranes seal the volatile liquid electrolyte. With recent advances in in situ electrochemical liquid cell TEM holders, it is now possible to image dynamically evolving electrochemical reactions while simultaneously performing quantitative electrochemical measurements [3-4]. Here we use an in situ liquid biasing flow-cell TEM holder (Hummingbird Scientific) within a Hitachi HF3300 S/TEM operating at $300 \mathrm{kV}$. The biasing microchips used in these experiments have either $\mathrm{Pt}$ or $\mathrm{Au}$ electrodes patterned onto the surface of a SiN membrane which allows for detailed investigation of SEI formation reaction mechanisms and kinetics onto and from the surface of the bare electrodes themselves or on focus ion beam (FIB) prepared electrodes. Here we have developed a method to perform quantitative in situ electrochemical measurements in a two or three-electrode cell configuration using metallic $\mathrm{Li}$ as a counter and reference electrode. To assemble the electrochemical cell, Li metal is interfaced with one or two of the electrical leads within the tip of the holder. The working electrode is interfaced with electrodes patterned onto the microchip. Liquid electrolyte is delivered to the cell using a microfluidic syringe pump and tubing at a flow rate of $5 \mu \mathrm{L} / \mathrm{min}$. During in situ TEM experiments, the flow rate is set at $1 \mu \mathrm{L} / \mathrm{min}$ to prevent the dewetting of the electrode and to transport out any products from the counter electrode. All specimen loading and electrochemical cell assembly is performed in an inert argon atmosphere glovebox. A potentiostat (BioLogic SP-200 equipped with a low current probe) is interfaced with the in situ TEM holder for electrochemical measurements.

In order to conduct more detailed and quantitative electrochemical studies the current or charge must be measured. Li as a reference electrode allows for the direct comparison of transient TEM images 
to bulk the electrochemical studies. Here we performed a variety of electrochemistry characterization methods applied specifically to study SEI formation mechanisms and kinetics on Au and $\mathrm{Si}$ electrodes. The organic Li-ion battery electrolytes were selected to include those that have practical use in industry (e.g. $\mathrm{LiPF}_{6}$ in $\mathrm{EC}: \mathrm{DMC}$ ). Preliminary results show that current may be measured during chronoamperometry and cyclic voltammetry (Fig. 1) with little noise $\sim \pm 0.01 \mathrm{nA}$. These cells possess high-uncompensated resistance due to the micron scaled electrode size and constrained current lines. Our two- or three-electrode setup is able to compensate for this resistance and provide more accurate working potentials. The observation of SEI formation and Li deposition on Au serves as a proof-of-concept in the utility and design of in situ TEM in the investigation of the electrode|electrolyte interface. SEI formation on Si will be discussed as well as relating charging rates to $\mathrm{Li}$ intercalation induced swelling of $\mathrm{Si}$ anodes. Formation of nanoparticles and dense polymeric regions in addition to the swelling of electrodes due to $\mathrm{Li}$ insertion will be related to potential and charge transfer rates. The quantitative in situ TEM based electrochemical characterization method developed here provides a new means to characterize the underpinnings of complex electrochemical reactions [5].

\section{References}

[1] E. Peled, Journal of the Electrochemical Society. 126[12], (1979), 2047-2051.

[2] P. Verma, et al, Electrochimica Acta. 55, (2010).6332-6341.

[3] M.J. Williamson, et al, Nature Materials. 2, (2003), 532-536.

[4] R.R. Unocic, et al, Microscopy and Microanalysis, 17 (Suppl 2), (2011), 1564-1565.

[5] Research supported by the Fluid Interface Reactions Structures and Transport (FIRST) Center, an Energy Frontier Research Center funded by the Office of Basic Energy Sciences (BES)-DOE. Microscopy performed as part of a user proposal at ORNL's Shared Research Equipment (ShaRE) User Facility, which is sponsored by BES-DOE.
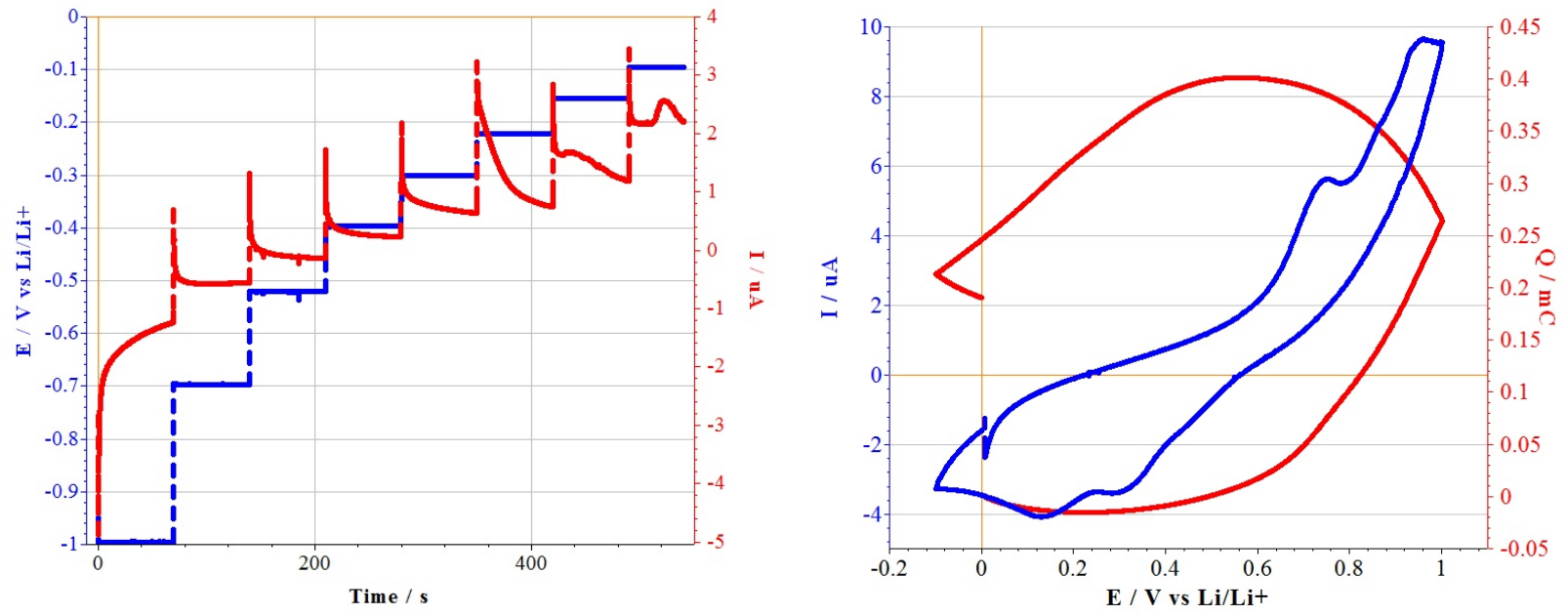

Figure 1. Chronoamperometry plot (left) and cyclic voltammogram (right) of Si FIB-ed onto Pt. Sweep rate: $5 \mathrm{mV} \mathrm{s}^{-1}$; electrolyte: $1 \mathrm{M} \mathrm{LiPF}_{6}$ in EC:DEC. 